\title{
Raccoons in San Diego County as Sentinels for West Nile Virus Surveillance
}

\author{
Sarah C. Marikos*1, Karen L. Ferran'1, Esmeralda Iniguez-Stevens ${ }^{1}$ and Nikos Gurfield ${ }^{2}$ \\ 'Early Warning Infectious Disease Surveillance Program (EWIDS), California Department of Public Health, San Diego, CA, USA; \\ ${ }^{2}$ County of San Diego, Department of Environmental Health, San Diego, CA, USA
}

\section{Objective}

To investigate the potential of utilizing raccoons as sentinels for West Nile Virus (WNV) in an effort to guide public health surveillance, prevention, and control efforts.

\section{Introduction}

Since its detection in 1999 in New York, WNV spread westward across the continent, and was first detected in California in 2003 in Imperial County (1). In California and in many states, birds, especially corvids, are used as sentinel animals to detect WNV activity. Recent seroprevalence studies have shown WNV activity in different wild mammalian species (1-3); in the United States, WNV seroprevalence in some studies in raccoons has ranged from $34-46 \%$ $(3,4)$. In addition, it has been shown that after experimental infection, raccoons can attain high viral titers and shed WNV in their saliva and feces (5). Given their peridomestic nature, we investigated the feasibility of their use as sentinels for early warning of WNV and as indicators of WNV activity as a strategy to better localize WNV transmission foci in guiding vector control efforts.

\section{Methods}

Sick, injured or orphaned raccoons undergoing rehabilitation at Project Wildlife, one of the largest, non-profit wildlife rehabilitation organizations in the United States, located in San Diego County, were tested for WNV shedding. Project Wildlife team members who regularly care for sick, injured, or orphaned raccoons were trained to collect oral and fecal samples for viral testing during 2011 and 2012 upon raccoons' arrival to Project Wildlife. Oral and fecal samples were tested using real-time PCR for the envelope gene of WNV.

\section{Results}

To date 71 raccoons have been tested for WNV and all PCR test results have been negative. Of the 71 raccoons tested from May 2011 to October 2011 and June 2012 to September 2012, 85.9\% ( $\mathrm{n}=61)$ had age classification data. The majority of these raccoons were young; $52.5 \%(n=32)$ were days or weeks old and $39.3 \%(n=24)$ were classified as juveniles. All raccoons were found primarily in urban settings at least 20 miles from the northern edge of the County.

\section{Conclusions}

While none of the raccoon samples tested in this study were found to be WNV positive, surveillance data from San Diego County suggests that WNV activity during this time period was extremely low. From January-October 2011, San Diego County Vector Control reported all negative results for WNV in dead birds, sentinel chickens, horses, and humans for WNV; only 1 mosquito pool from the north- ern border region of the County tested positive for WNV (6). Thus, despite WNV activity throughout the state of California, the virus did not appear to be circulating widely in San Diego County in 2011 (7). To date during the 2012 season, San Diego County reported all negatives for WNV in dead birds, sentinel chickens, mosquito pools, and horses; only one human case of WNV was identified in an asymptomatic male during a routine blood donation (6).

Further evaluation is needed to determine if raccoons are useful sentinel species for WNV surveillance. Testing should continue to evaluate if raccoons may serve as a more effective early warning sentinel for WNV than birds which can travel long distances from the exposure site, and to determine if raccoons may allow better localization of WNV activity.

\section{Keywords}

West Nile Virus; Early warning surveillance; Raccoons; Sentinels

\section{Acknowledgments}

The authors acknowledge Project Wildlife staff and volunteers who generously donated their time to this study, and who provide the utmost quality care for sick, injured, or orphaned raccoons.

\section{References}

1 Reisen, W, Lothrop H, Chiles R, Madon M, Cossen C, Woods, L, et al. West Nile in California. Emerg Infect Dis. 2004;10:1369.

2 Docherty, DE, Samuel, MD, Nolden, CA, Egstad, KF, Griffin, KM. West Nile Virus Antibody Prevalence in Wild Mammals, Southern Wisconsin. Emerg Infect Dis. 2006;12:1982.

3 Bentler, KT, Hall, JS, Root, JJ, Klenk, K, Schmit, B, Blackwell, BF. Serologic Evidence of West Nile Virus Exposure in North American Mesopredators. 2007;76:173.

4 Blitvich, BJ, Juarez, LI, Tucker, BJ, Rowley, WA, Platt, KB. Antibodies to West Nile Virus in Raccoons and Other Wild Peridomestic Mammals in Iowa. 2009;45:1163.

5 Root, JJ, Bentler, KT, Nemeth, NM, Gidlewski, T, Spraker, TR, Franklin, AB. Experimental Infection of Raccoons (Procyon lotor) with West Nile Virus. Am J Trop Med Hyg. 2010;83:803.

6 County of San Diego, Department of Environmental Health, "West Nile Cases in San Diego County", accessed September 5, 2012.

7 California Department of Public Health, "West Nile Page", accessed September 5, 2012.

*Sarah C. Marikos

E-mail: smarikos@cdph.ca.gov 